\title{
From ground states to local Hamiltonians
}

\author{
Jianxin Chen, ${ }^{1,2}$ Zhengfeng Ji, ${ }^{2,3}$ Bei Zeng, ${ }^{1,2}$ and D. L. Zhou ${ }^{4}$ \\ ${ }^{1}$ Department of Mathematics and Statistics, University of Guelph, Guelph, Ontario, Canada \\ ${ }^{2}$ Institute for Quantum Computing, University of Waterloo, Waterloo, Ontario, Canada \\ ${ }^{3}$ State Key Laboratory of Computer Science, Institute of Software, Chinese Academy of Sciences, Beijing, China \\ ${ }^{4}$ Beijing National Laboratory for Condensed Matter Physics and Institute of Physics, Chinese Academy of Sciences, Beijing 100190, China
}

(Received 13 November 2011; published 30 August 2012)

\begin{abstract}
Traditional quantum physics solves ground states for a given Hamiltonian, while quantum information science asks for the existence and construction of certain Hamiltonians for given ground states. In practical situations, one would be mainly interested in local Hamiltonians with certain interaction patterns, such as nearest-neighbor interactions on some types of lattices. A necessary condition for a space $V$ to be the ground-state space of some local Hamiltonian with a given interaction pattern is that the maximally mixed state supported on $V$ is uniquely determined by its reduced density matrices associated with the given pattern, based on the principle of maximum entropy. However, it is unclear whether this condition is in general also sufficient. We examine the situations for the existence of such a local Hamiltonian to have $V$ satisfying the necessary condition mentioned above as its ground-state space by linking to faces of the convex body of the local reduced states. We further discuss some methods for constructing the corresponding local Hamiltonians with given interaction patterns, mainly from physical points of view, including constructions related to perturbation methods, local frustration-free Hamiltonians, as well as thermodynamical ensembles.
\end{abstract}

DOI: 10.1103/PhysRevA.86.022339

PACS number(s): 03.67.Mn, 03.65.Ud, 89.70.Cf

\section{INTRODUCTION}

Traditional quantum many-body physics focuses on finding ground-state energy and the corresponding ground states for some given Hamiltonians. A naturally occurring Hamiltonian involves only one- and two-body interactions in most cases. The new field of quantum information science, however, focuses more on studying quantum states [1]. Quantum states are "information carriers" of quantum information upon which communication is conveyed and computation is implemented. After years of development, it has become clear that quantum communication and computation offers the possibility of a secure and high rate of information transmission and fast computational solution of certain important problems, which is at the heart of modern information technology.

One major direction of quantum information science is to study correlations in many-body quantum systems. Here the term correlation is used instead of entanglement due to the fact that a quantum state contains both classical and quantum correlation, which both contribute to real physical phenomena. Traditionally, correlation is characterized by correlation functions, which are directly related to experimental measurements of physical observables. Quantum information science brings new angles to study correlations from information-scientific points of view.

An interesting viewpoint on correlation in quantum states is based on the principle of maximum entropy, which is advocated by Jaynes in the study on the foundation of statistical mechanics [2]. The principle says that if an $n$-particle quantum state $\rho$ has the maximum entropy among all the $n$-particle states with the same $k$-particle reduced density matrices $(k$ RDMs) as those of $\rho$, then $\rho$ contains no more information than that contained in its $k$-RDMs. And such a $\rho$ consistent with the given $k$-RDMs is indeed unique. In this sense, $\rho$ contains no irreducible $r$-particle correlation for any $r>k[3,4]$.
In the case that $\rho$ is a pure state, $\rho$ is uniquely determined by its $k$-RDMs based on the principle of maximum entropy. It simply means that there does not exist any other state, pure or mixed, which has the same $k$-RDMs as those of $\rho$. Well-known examples include: almost all three-qubit pure states are uniquely determined by their 2-RDMs [5], almost every pure state of many-body quantum systems (with equal dimensional subsystems) is uniquely determined by its RDMs of just over half of the parties $[3,6], W$-type states are uniquely determined by their 2-RDMs [7], and the only $n$-particle pure states which cannot be determined by their $(n-1)$ RDMs are those Greenberger-Horne-Zeilinger (GHZ)-type states [8].

A many-body Hamiltonian $H$ is $k$ local if $H=\sum_{i} H_{i}$, where each term $H_{i}$ acts nontrivially on, at most, $k$ particles. In practical situations, one would be mainly interested in $k$ local Hamiltonians with certain interaction patterns, such as nearest-neighbor interactions on some type of lattices. That is, for a given space $V$, one would like to know whether $V$ can be the ground-state space of some $k$-local Hamiltonian $H=\sum_{i} H_{i}$, which contains only certain terms of $k$-particle interactions, and, if such a $k$-local Hamiltonian exists, how to find it.

In this paper, we address this question by starting from a natural necessary condition for a space $V$ to be the ground-state space of some local Hamiltonian with a given interaction pattern. That is, the maximally mixed state supported on $V$ is uniquely determined by its reduced density matrices associated with the given interaction pattern, based on the principle of maximum entropy. This condition builds an interesting link between correlations of quantum states and ground-state spaces of local Hamiltonians; unfortunately, it is unclear whether this condition is in general also sufficient. We examine the situations for the existence of such a local Hamiltonian to have $V$ satisfying the necessary condition mentioned above as 
its ground-state space by linking to faces of the convex body of the local reduced states. We then further discuss some methods for constructing a corresponding $k$-local Hamiltonian, mainly from physical points of view, including constructions related to perturbation methods, local frustration-free Hamiltonians, as well as thermodynamical ensembles.

We organize our paper as follows. In Sec. II, we give a formal definition of local Hamiltonians of a given interaction pattern and review the convex geometry viewpoint of their ground-state spaces. In Sec. III, we introduce the concept of $\mathcal{K}$-correlated subspaces and link it to the correlation of ground-state spaces of local Hamiltonians and discuss its meaning in terms of convex geometry. In Sec. IV, we examine in more detail the situations where a $\mathcal{K}$-correlated subspace may fail to be the ground-state space of the corresponding local Hamiltonian of a given interaction pattern, and provide a perturbation method to construct such a Hamiltonian if it exists. In Sec. V, we provide another method of finding the local Hamiltonians of some frustrated systems starting from some frustration-free systems, which combined with the perturbation method succeeds in finding the local Hamiltonians in certain special cases. For instance, this allows us to identify Hamiltonians for almost all three-qubit states and the $n$-qubit $W$ states with only nearest-neighbor interactions on a one-dimensional spin chain. In Sec. VI, we provide a general method of finding the local Hamiltonians from a thermal ensemble idea. Finally, a summary and discussion is given in Sec. VII.

\section{LOCAL HAMILTONIANS AND CONVEX GEOMETRY}

This section discusses the ground-state space properties of local Hamiltonians. We start from a formal discussion of local Hamiltonians with given interaction patterns.

Consider an $n$-particle system. We specify a pattern $\mathcal{K}$, where each element $K_{j} \in \mathcal{K}$ is a subset of $\{1,2, \ldots, n\}$ with $\left|K_{j}\right|=k$ (here, $\left|K_{j}\right|$ is the size of $K_{j}$ ). A Hamiltonian $H=$ $\sum_{i} H_{i}$ is called $\mathcal{K}$ local if each $H_{i}$ acts nontrivially on, at most, $k$ particles in some $K_{j} \in \mathcal{K}$. In practice, the choice of such a pattern $\mathcal{K}$ is usually related to certain spatial geometry considerations, such as nearest-neighbor particles with respect to some spin lattices.

As an example, the Hamiltonian $H$ of three qubits,

$$
H=J\left(X_{1} X_{2}+X_{2} X_{3}\right)+B\left(Z_{1}+Z_{2}+Z_{3}\right),
$$

is $\mathcal{K}$ local where

$$
\mathcal{K}=\{\{1,2\},\{2,3\}\} .
$$

Here, $X_{j}, Y_{j}, Z_{j}$ are Pauli $X, Y, Z$ operators, respectively, acting on the $j$ th qubit.

Note that for any $\tilde{\mathcal{K}} \supseteq \mathcal{K}$, a Hamiltonian $H$ that is $\mathcal{K}$ local is also $\tilde{\mathcal{K}}$ local. Furthermore, for some $k^{\prime} \geqslant k$ and a pattern $\mathcal{K}^{\prime}$ with $\left|K_{j}^{\prime}\right| \leqslant k^{\prime}, H$ is also $\mathcal{K}^{\prime}$ local if for any $K_{i} \in \mathcal{K}$ there exists some $K_{j}^{\prime} \in \mathcal{K}^{\prime}$ such that $K_{i} \subseteq \mathcal{K}_{j}^{\prime}$. For instance, the Hamiltonian given in Eq. (1) is also $\{\{1,2\},\{2,3\},\{1,3\}\}$ local or $\{\{1,2,3\}\}$ local. In practice, we would usually be interested in the smallest number $k$ and the smallest possible set $\mathcal{K}$ such that $H$ is $\mathcal{K}$ local.

Let $\mathcal{D}$ be the set of density matrices of $n$ particles. For any given pattern $\mathcal{K}$, list all of the elements $K_{i} \in \mathcal{K}$ as a vector
$\left(K_{1}, K_{2}, \ldots, K_{M}\right)$ in a fixed order, where $M$ is the size of $\mathcal{K}$. When $M=\left(\begin{array}{l}n \\ k\end{array}\right), \mathcal{K}$ contains all of the $k$-element subset of $\{1,2, \ldots, n\}$. Let $\gamma_{K_{i}}$ be the $k$-RDM of particles in $K_{i} \in \mathcal{K}$. For $\rho \in \mathcal{D}$, let

$$
\vec{R}_{\mathcal{K}}(\rho)=\left(\gamma_{K_{1}}, \gamma_{K_{2}}, \ldots, \gamma_{K_{M}}\right),
$$

which is a vector whose elements are $k$-RDMs of $\rho$.

Note a simple fact that the set

$$
\mathcal{D}_{\mathcal{K}}=\left\{\vec{R}_{\mathcal{K}}(\rho) \mid \rho \in \mathcal{D}\right\}
$$

is a closed convex set. Indeed, it has been known that there is a natural connection between ground-state spaces and exposed faces of the convex set $\mathcal{D}_{\mathcal{K}}$ (see, for instance, $[9,10]$ ), which we briefly review here.

We first recall some notations from convex analysis. For a convex set $C$, its dual cone $\mathcal{P}(C)$ is

$$
\mathcal{P}(C)=\{\vec{y} \mid \forall \vec{x} \in C,\langle\vec{x}, \vec{y}\rangle \geqslant 0\} .
$$

Let the dual cone of $\mathcal{D}_{\mathcal{K}}$ be $\mathcal{P}_{\mathcal{K}}$. For the vectors $\vec{x}=$ $\left(\gamma_{1}, \gamma_{2}, \ldots, \gamma_{M}\right)$ and $\vec{y}=\left(H_{1}, H_{2}, \ldots, H_{M}\right)$ with Hermitian $H_{j} \mathrm{~s},\langle\vec{x}, \vec{y}\rangle$ is defined as $\sum_{j=1}^{M} \operatorname{Tr}\left(H_{j} \gamma_{j}\right)$. Any point $\vec{H}$ of the form $\left(H_{1}, H_{2}, \ldots, H_{M}\right)$ defines a $\mathcal{K}$-local Hamiltonian $H=$ $\sum_{j=1}^{M} H_{j}$. Moreover, we have $\left\langle\vec{R}_{\mathcal{K}}(\rho), \vec{H}\right\rangle=\operatorname{Tr}(\rho H)$. This allows us to visualize $\mathcal{K}$-local Hamiltonians as hyperplanes in the space containing $\mathcal{D}_{\mathcal{K}}$. More specifically, let $\vec{H}$ be a point that corresponds to the Hamiltonian $H$ and define a hyperplane also denoted as $H$ to be

$$
H=\{\vec{x} \mid\langle\vec{x}, \vec{H}\rangle=0\} .
$$

For any convex set $C$, a subset $F$ is called a face on $C$ if

(1) $F$ is a convex set, and

(2) for any line segment $L \subseteq C$, if $L$ intersects $F$ at some point other than the two end points of $L$, then $L \subseteq F$ [11].

A face $F$ is exposed if there exists some element $\vec{y}$ in the dual cone $\mathcal{P}(C)$ such that $\langle\vec{x}, \vec{y}\rangle=0, \forall \vec{x} \in F$ and $\langle\vec{x}, \vec{y}\rangle\rangle$ $0, \forall \vec{x} \notin F$.

Let the set $F_{V}$ be the image in $\mathcal{D}_{\mathcal{K}}$ for the states supported on the space $V$; that is,

$$
F_{V}=\left\{\vec{R}_{\mathcal{K}}(\sigma) \mid \operatorname{range}(\sigma) \subseteq V\right\} .
$$

Then, for any $V$ that is a ground-state space of some $\mathcal{K}$-local Hamiltonian, $F_{V}$ is an exposed face of $\mathcal{D}_{\mathcal{K}}$.

\section{III. $\mathcal{K}$-CORRELATED SPACES}

For any $n$-particle quantum state $\rho$, define a set $A_{\mathcal{K}}(\rho)$ of $n$-particle quantum states which have the same array of $k$-RDMs as $\rho$, i.e.,

$$
A_{\mathcal{K}}(\rho)=\left\{\sigma \in \mathcal{D} \mid \vec{R}_{\mathcal{K}}(\sigma)=\vec{R}_{\mathcal{K}}(\rho)\right\}
$$

Let $\tilde{\rho}_{\mathcal{K}}$ denote the state of maximum entropy among all the states in $A_{\mathcal{K}}(\rho)$, i.e.,

$$
\tilde{\rho}_{\mathcal{K}}=\operatorname{argmax}\left\{S(\sigma) \mid \sigma \in A_{\mathcal{K}}(\rho)\right\},
$$

where the von Neumann entropy $S(\rho)=-\operatorname{Tr}\left(\rho \log _{2} \rho\right)$. Note that $\tilde{\rho}_{\mathcal{K}}$ is indeed unique. 
Based on the principle of maximum entropy, $\tilde{\rho}_{\mathcal{K}}$ contains no more information than is contained in the reduced density matrices $\gamma_{K_{i}} s$. Therefore, if $\rho=\tilde{\rho}_{\mathcal{K}}$, then $\rho$ is the state containing no more information than is contained in the reduced density matrices $\gamma_{K_{i}} s$. In other words, $\rho$ can be determined without ambiguity from $\gamma_{K_{i}}$ s. In this sense, we say that the state $\rho$ is uniquely determined by $\gamma_{K_{i}} s$, and call it $\mathcal{K}$ correlated. That is, an $n$-particle state $\rho$ is called $\mathcal{K}$ correlated if $\rho=\tilde{\rho}_{\mathcal{K}}$.

As an example for $\mathcal{K}$-correlated states, consider the threequbit state

$$
\rho_{c}=\frac{1}{2}(|000\rangle\langle 000|+| 111\rangle\langle 111|) .
$$

For $\mathcal{K}=\{\{1,2\},\{2,3\}\}$, it is straightforward to check that among all the three-qubit states with the same 2-RDMs for particles $\{1,2\}$ and $\{2,3\}, \rho_{c}$ has the maximum entropy. So $\rho_{c}$ is $\mathcal{K}$ correlated. On the other hand, the three-qubit GHZ state

$$
|\mathrm{GHZ}\rangle=\frac{1}{\sqrt{2}}(|000\rangle+|111\rangle)
$$

has the same 2-RDMs for particles $\{1,2\}$ and $\{2,3\}$ as $\rho_{c}$, but $\rho_{c}$ has a larger von Neumann entropy than that of $|\mathrm{GHZ}\rangle$. Therefore, $|\mathrm{GHZ}\rangle$ is not $\mathcal{K}$ correlated.

Note that similar to the case of $\mathcal{K}$-local Hamiltonians, for any $\tilde{\mathcal{K}} \supseteq \mathcal{K}$, a state $\rho$ that is $\mathcal{K}$ correlated is also $\tilde{\mathcal{K}}$ correlated. Furthermore, for some $k^{\prime}>k$ and a pattern $\mathcal{K}^{\prime}$ with $\left|K_{j}^{\prime}\right|=k^{\prime}$, $\rho$ is also $\mathcal{K}^{\prime}$ correlated if for any $K_{i} \in \mathcal{K}$ there exists some $K_{j}^{\prime} \in \mathcal{K}^{\prime}$ such that $K_{i} \subseteq \mathcal{K}_{j}^{\prime}$. In practice, for a given $\rho$, we usually would like to find the smallest possible number $k$ and the smallest possible set $\mathcal{K}$ such that $\rho$ is $\mathcal{K}$ correlated.

For a space $V$, if the maximally mixed state $\rho_{V}$ supported on $V$ is $\mathcal{K}$ correlated, then we call the space $\mathcal{K}$ correlated. The following simple observation then links the ground-state space of $\mathcal{K}$-local Hamiltonians and $\mathcal{K}$-correlated space.

Observation 1. If $V$ is the ground-state space of some $\mathcal{K}$ local Hamiltonian, then $V$ is $\mathcal{K}$ correlated.

This is because for any state $\rho$ supported on $V, \operatorname{Tr}(\rho H)$ equals the ground energy. Then obviously the maximally mixed state $\rho_{V}$ supported on $V$ has the maximum entropy among all states in $A_{\mathcal{K}}(\rho)$.

In the case of pure states, that is, where $V$ is onedimensional, Observation 1 states that a necessary condition for a pure state $|\psi\rangle$ to be a unique ground state of some $\mathcal{K}$-local Hamiltonian is that $|\psi\rangle$ is uniquely determined by its $k$-RDMs of particles in all $K_{j} \in \mathcal{K}$.

As a simple example, consider the one-dimensional space $V$ which is spanned by the three-qubit GHZ state, given by Eq. (10). Because $\rho_{V}$ is not $\mathcal{K}$ correlated as discussed in a previous example, there does not exist a $\mathcal{K}$-local Hamiltonian whose unique ground state is $|\mathrm{GHZ}\rangle$.

Observation 1 tells us that in order to find the desired $\mathcal{K}$ local Hamiltonian for a given space $V$, first of all $V$ must be $\mathcal{K}$ correlated. Therefore a $\mathcal{K}$-correlated space is then a natural starting point for talking about the general problem of "from ground states to local Hamiltonians."

One would then wonder whether the necessary condition of $\mathcal{K}$ correlatedness for a space $V$ being a ground-state space of some $\mathcal{K}$-local Hamiltonian is also sufficient, which indeed gives rise to the main question we will discuss in this paper, as stated below.
Main Question: Given a $\mathcal{K}$-correlated space $V$, does there exist a $\mathcal{K}$-local Hamiltonian which has $V$ as its groundstate space, and, if yes, then how can we construct such a Hamiltonian?

Unfortunately, this question seems difficult to answer in general. In seeking a better understanding, we start by examining a helpful property of $\mathcal{K}$-correlated spaces, given by the following observation.

Observation 2. For a $\mathcal{K}$-correlated space $V$ and any state $\rho$ supported on $V$, any state $\sigma$ in $A_{\mathcal{K}}(\rho)$ is also supported on $V$.

To see why this is the case, denote the range of $\rho$ by range $(\rho)$, which is the space spanned by all of the eigenstates of $\rho$ with nonzero eigenvalues. Since $V$ is $\mathcal{K}$ correlated, we know that the maximally mixed state $\rho_{V}$ supported on $V$ satisfies $\tilde{\rho}_{V, \mathcal{K}}=\rho_{V}$. Therefore, $\operatorname{range}(\hat{\rho}) \subseteq \operatorname{range}\left(\rho_{V}\right)$ for any $\hat{\rho} \in A_{\mathcal{K}}\left(\rho_{V}\right)$. Now for any $\rho$ supported on $V$, we have range $(\rho) \subseteq \operatorname{range}\left(\rho_{V}\right)$. Consequently, for any $\sigma \in A_{\mathcal{K}}(\rho)$, we have range $(\sigma) \subseteq \operatorname{range}\left(\rho_{V}\right)$, meaning that $\sigma$ is also supported on $V$. Note that for this argument, there are indeed some subtle points that need to be clarified. We then include a complete proof of this observation in the Appendix.

Next, we build a connection between $\mathcal{K}$-correlated spaces and faces of the convex set $\mathcal{D}_{\mathcal{K}}$, which is given by the following observation.

Observation 3. For a $\mathcal{K}$-correlated space $V, F_{V}$ is a face of the convex set $\mathcal{D}_{\mathcal{K}}$.

To show that this observation holds, first note that it is obvious that $F_{V}$ is a convex set. Then for two states $\rho_{0}$ and $\rho_{1}$, let $L$ be a line segment in $\mathcal{D}_{\mathcal{K}}$ with end points $\vec{R}_{\mathcal{K}}\left(\rho_{0}\right)$ and $\vec{R}_{\mathcal{K}}\left(\rho_{1}\right)$. If $L$ intersects $F_{V}$ at a point $(1-p) \vec{R}_{\mathcal{K}}\left(\rho_{0}\right)+$ $p \vec{R}_{\mathcal{K}}\left(\rho_{1}\right)$ for some $p \in(0,1)$, then $\exists \sigma$ is supported on $V$ such that $(1-p) \rho_{0}+p \rho_{1} \in A_{\mathcal{K}}(\sigma)$. When $V$ is $\mathcal{K}$ correlated, we have

$$
\text { range }\left[(1-p) \rho_{0}+p \rho_{1}\right] \subseteq V,
$$

and therefore both range $\left(\rho_{j}\right)$ are spaces of $V$. It then follows that the entire line segment $L$ is in $F_{V}$.

Note that it is straightforward to show that the reverse of this observation is also true. That is, for any face $F_{V}$ of $\mathcal{D}_{\mathcal{K}}, V$ is $\mathcal{K}$ correlated.

Observation 3 characterizes the image $F_{V}$ in $\mathcal{D}_{\mathcal{K}}$ of a $\mathcal{K}$ correlated space $V$ as a face of the convex set $\mathcal{D}_{\mathcal{K}}$. And we know that ground-state spaces of $\mathcal{K}$-local Hamiltonians correspond to exposed faces of $\mathcal{D}_{\mathcal{K}}$. Therefore, the question of whether a $\mathcal{K}$-local Hamiltonian exists to have the given $\mathcal{K}$-correlated space as its ground-state space then becomes the determination of whether the corresponding face $F_{V}$ is exposed in $\mathcal{D}_{\mathcal{K}}$. We examine this question further in the next section.

\section{NONEXPOSED FACES}

We know that for a general convex set $C$, there do exist nonexposed faces. An example is shown in Fig. 1. However, for a given interaction pattern $\mathcal{K}$, the geometry of $\mathcal{D}_{\mathcal{K}}$ is in general difficult to analyze. Indeed, we know that for local Hamiltonian problems of practical interest, even with the existence of a quantum computer, the membership of $\mathcal{D}_{\mathcal{K}}$ is very difficult to determine [12].

Here we just try to get a bit further to analyze an artificial example. We consider a two-qubit system. In this case, instead 


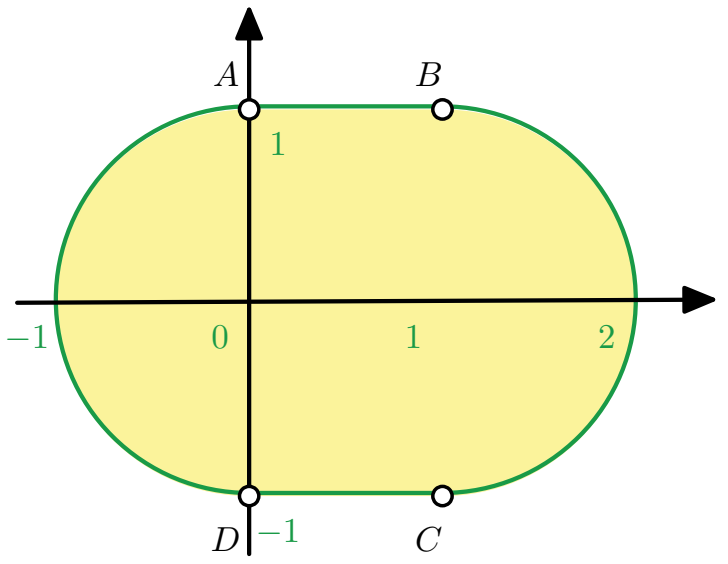

FIG. 1. (Color online) The convex set of points given by $[x=$ $\left.\operatorname{Tr}\left(\rho H_{1}\right), y=\operatorname{Tr}\left(\rho H_{2}\right)\right]$. The convex set is the union of two half disks on the left and right and a rectangle in the middle. Points $A, B, C, D$ are by definition faces of this convex set, yet for each point there is no line that touches the convex set only at the point.

of only requiring a $\mathcal{K}$-local Hamiltonian, we want a $\mathcal{K}$-local Hamiltonian of a certain type. More precisely, we want a onebody Hamiltonian $H$ which can only have local terms of $H_{1}$ and $\mathrm{H}_{2}$, as given below:

$$
H_{1}=X_{2}+\frac{1}{2}\left(I+Z_{1}\right), \quad H_{2}=Y_{2} .
$$

Now, for any given two-qubit state $|\psi\rangle$ which can be uniquely determined by its mean values on $H_{1}$ and $H_{2}$, we wonder whether there exists a Hamiltonian $H_{\psi}=\alpha H_{1}+\beta H_{2}$ that has $|\psi\rangle$ as its unique ground state. Note that in this case, such a $|\psi\rangle$ is a natural analog of a $\mathcal{K}$-correlated state and it corresponds to an extreme point of the two-dimensional convex set given by all points of $\left[x=\operatorname{Tr}\left(\rho H_{1}\right), y=\operatorname{Tr}\left(\rho H_{2}\right)\right]$, where $\rho$ is any two-qubit quantum state. This convex set is shown in Fig. 1.

What we can see from Fig. 1 is that there are four nonexposed extreme points $A, B, C, D$ [with coordinates $(0,1),(1,1),(1,-1),(0,-1)]$. If we denote $\left|0_{y}\right\rangle,\left|1_{y}\right\rangle$ the eigenstates of $Y$ with eigenvalues \pm 1 , respectively, then these four nonexposed extreme points correspond to quantum states $|0\rangle\left|0_{y}\right\rangle,|1\rangle\left|0_{y}\right\rangle,|1\rangle\left|1_{y}\right\rangle,|0\rangle\left|1_{y}\right\rangle$, respectively. For each of these four states, apparently it cannot be a unique ground state of any kind of Hamiltonian with terms of $H_{1}$ and $H_{2}$ only, as $|0\rangle\left|0_{y}\right\rangle$ must always be degenerate with $|1\rangle\left|0_{y}\right\rangle$, and $|0\rangle\left|1_{y}\right\rangle$ must be degenerate with $|1\rangle\left|1_{y}\right\rangle$.

This example is somewhat artificial as one can certainly find a one-body Hamiltonian which has, for instance, $|0\rangle\left|0_{y}\right\rangle$ as its unique ground state if we do not restrict on the terms of $H_{1}$ and $\mathrm{H}_{2}$ only. However, it is unclear whether such a relaxation to allow any $\mathcal{K}$-local terms is enough to remove all nonexposed faces in general. The answer would require deeper physical insight beyond a general geometric analysis of these restricted kinds of Hamiltonians. On the other hand, in practice, there might also be physical situations which restrict the form of the terms appearing in a $\mathcal{K}$-local Hamiltonian (e.g., symmetry restrictions), where a nonexposed face situation might possibly arise.

In practice, for a given $\mathcal{K}$-correlated space $V$, we may circumvent the "existence analysis" and nevertheless go ahead

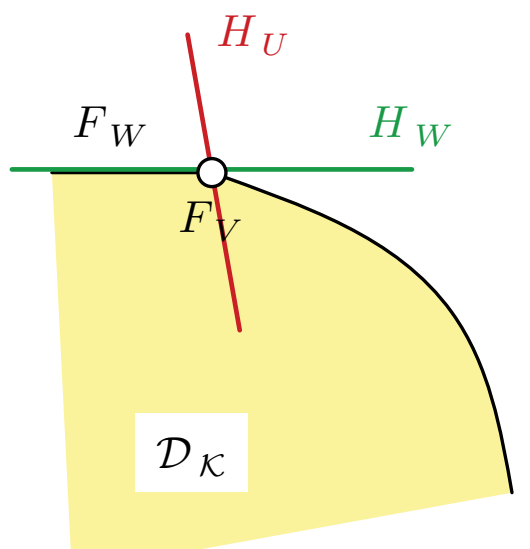

FIG. 2. (Color online) $H_{W}$ is a line that touches the convex set $\mathcal{D}_{\mathcal{K}}$ at the top line segment $F_{W}$, while $H_{U}$ goes through point $F_{V}$, but does not contain points in $F_{W} \backslash F_{V}$.

and try to construct the corresponding $\mathcal{K}$-local Hamiltonian. The geometric viewpoint of exposed and nonexposed faces does give some clue of how to do that. We then discuss a method of perturbation of finding a $\mathcal{K}$-local Hamiltonian $H$ for a given $\mathcal{K}$-correlated space $V$ based on this geometric point of view, in case there indeed exists such an $H$.

An illustration of the idea is given in Fig. 2. For a given $\mathcal{K}$-correlated space $V$, our goal is to find some $\mathcal{K}$-local Hamiltonian $H$ such that the ground space of $H$ is exactly $V$. As we have already mentioned, this is equivalent to finding some point $\vec{H}$ in $\mathcal{P}_{\mathcal{K}}$ such that $H \bigcap \mathcal{D}_{\mathcal{K}}=F_{V}$, where $H$ is the hyperplane defined by $\vec{H}$. As $F_{V}$ is a face of $\mathcal{D}_{\mathcal{K}}$, we can find a hyperplane that contains $F_{V}$. Let this hyperplane be $H_{W}$ and a corresponding point in $\mathcal{P}_{\mathcal{K}}$ be $\vec{H}_{W}$, where $W$ is the ground space of the corresponding Hamiltonian $H_{W}$. We know that $W$ is also $\mathcal{K}$ correlated, as $H_{W}$ is a $\mathcal{K}$-local Hamiltonian. If $W$ equals $V$, then we are done. Otherwise, one sees that the intersection of hyperplane $H_{W}$ and $\mathcal{D}_{\mathcal{K}}$ is exactly the face $F_{W}$. Moreover, $F_{V}$ is a face of $F_{W}$.

Now we wish to find a perturbation $\mathcal{K}$-local Hamiltonian $H_{U}$ which can "split" the energy of states supported on $V$ and those supported on $W \backslash V$, where the Hamiltonian $H=$ $t H_{W}+H_{U}$ can have $V$ as its exact ground-state space for large-enough $t$. We show that the following conditions for $H_{U}$ are sufficient:

(1) $F_{V}$ is in the kernel of $H_{U}$.

(2) $\left\langle\vec{R}_{\mathcal{K}}(\rho), \vec{H}_{U}\right\rangle>0$ for all $\overrightarrow{\vec{R}}_{\mathcal{K}}(\rho) \in F_{W} \backslash F_{V}$.

Once there exists an $H_{U}$ that satisfies these two conditions, we can show that the Hamiltonian $H=t H_{W}+H_{U}$ can have $V$ as its exact ground-state space for large enough $t$. Let $U$ be the kernel of $H_{U}$. Denote $\lambda, \mu$ as the smallest positive eigenvalue of $H_{W}$ and $H_{U}$, respectively, and $\omega=\left\|H_{U}\right\|$ as the operator norm of $H_{U}$. For any $n$-particle state $|\psi\rangle$, write it as $\sum_{j=1}^{3}\left|\psi_{j}\right\rangle$ such that $\left|\psi_{1}\right\rangle \in U \cap W,\left|\psi_{2}\right\rangle \in W \cap U^{\perp}$, and $\left|\psi_{3}\right\rangle \in W^{\perp}$. Therefore, we have

$$
\begin{aligned}
& \left\langle\psi\left|\left(t H_{W}+H_{U}\right)\right| \psi\right\rangle \\
& \quad=t\left\langle\psi_{3}\left|H_{W}\right| \psi_{3}\right\rangle+\left(\left\langle\psi_{2}\left|+\left\langle\psi_{3}\right|\right) H_{U}\left(\left|\psi_{2}\right\rangle+\left|\psi_{3}\right\rangle\right)\right.\right. \\
& \quad \geqslant(t \lambda-\omega)\left\|\psi_{3}\right\|^{2}-2 \omega\left\|\psi_{2}\right\|\left\|\psi_{3}\right\|+\mu\left\|\psi_{2}\right\|^{2} .
\end{aligned}
$$


The above equation is non-negative if $\left\|\psi_{3}\right\|=0$. Otherwise, it is a quadratic function and is positive for large-enough $t$. This means that the ground space of $t H_{W}+H_{U}$ is $W \cap U=V$.

In general, we do not know whether such an $H_{U}$ does exist. However, as we will show in Sec. V, in practical situations, this method of perturbation can indeed help us find a $\mathcal{K}$-local $H$ for a given $\mathcal{K}$-correlated space $V$, in case there indeed exists such an $H$. On the other hand, if one fails to find such an $H_{U}$, then this indicates the existence of a nonexposed face.

Finally, we mention another direct application of the geometric viewpoint of $\mathcal{K}$-correlated spaces, given by the following observation.

Observation 4. The nontrivial intersection of two $\mathcal{K}$ correlated spaces $V_{1}$ and $V_{2}$ is $\mathcal{K}$ correlated. Furthermore, for two spaces $V_{1}$ and $V_{2}$ which are ground-state spaces of the $\mathcal{K}$-local Hamiltonians $H_{1}$ and $H_{2}$, respectively, the nontrivial intersection of $V_{1}$ and $V_{2}$ is a ground space of some other $\mathcal{K}$-local Hamiltonian.

To show why this is true, simply note that the intersection of two faces $F_{V_{1}}$ and $F_{V_{2}}$ of $\mathcal{D}_{\mathcal{K}}$ is another face $F_{V}$, where $V=V_{1} \cap V_{2}$. And because $F_{V}$ is the intersection of two faces, one can use the method of perturbation to find the $\mathcal{K}$-local Hamiltonian for $F_{V}$, in which the existence of $H_{U}$ is ready to verify. Indeed, the Hamiltonian which has $V$ as its exact ground-state space can be simply chosen as $\mathrm{H}_{1}+\mathrm{H}_{2}$.

\section{FROM FRUSTRATION-FREE TO FRUSTRATED SYSTEMS}

In this section, we discuss a method of finding a corresponding $\mathcal{K}$-local Hamiltonian for some given $\mathcal{K}$-correlated spaces, which is related to local frustration-free Hamiltonians. This will allow us to find the desired Hamiltonians for some spaces whose correlation patterns are well known, including the three-qubit pure states (the topic of Sec.V A), and the $n$-qubit $W$-type states (the topic of Sec.V B).

Given a space $V$ and a pattern $\mathcal{K}$, let $\rho_{V}$ be the maximally mixed state of $V$, and denote $\rho_{V}\left(K_{i}\right)$ as the $k$-RDMs of $\rho_{V}$ for particles in $K_{i} \in \mathcal{K}$. Let $V_{K_{i}}=\operatorname{range}\left(\rho_{V}\left(K_{i}\right)\right)$. If

$$
V=\bigcap_{K_{i} \in \mathcal{K}} V_{K_{i}} \otimes I_{\bar{K}_{i}}
$$

where $\bar{K}_{i}=\{1,2, \ldots, n\} \backslash K_{i}$, then $V$ is the ground-state space of the $\mathcal{K}$-local Hamiltonian

$$
H=\sum_{K_{i} \in \mathcal{K}} H_{K_{i}},
$$

where $H_{K_{i}}$ is the projection onto the kernel of $\rho_{V}\left(K_{i}\right)$.

The Hamiltonian $H$ is known to be frustration free, as the ground-state space $V$ of $H$ is also the ground-state space of each term in the summation. That is, $V_{K_{i}}$ is the ground-state space of $H_{K_{i}}$. We call this kind of $\mathcal{K}$-local Hamitonian $\mathcal{K}$ frustration free $(\mathcal{K}$-FF). By Observation 1 , we know that the ground-state space $V_{\mathcal{K}}$ of a $\mathcal{K}$-FF Hamiltonian must be $\mathcal{K}$ correlated.

Although in general whether a space $V$ is the ground-state space of a $\mathcal{K}$-FF Hamiltonian could be difficult to analyze; that is, Eq. (12) is not easy to check for large systems. At least in principle, this provides a way of characterizing these kind of spaces as well as finding the corresponding $\mathcal{K}$-FF
Hamiltonians. This can then be used as a starting point to find a $\mathcal{K}$-local Hamiltonian for a space $V^{\prime} \subset V$ that is known to be $\mathcal{K}$ correlated, using the perturbation method discussed in Sec. II. The idea is, we choose the unperturbed Hamiltonian $H_{0}$ as the $\mathcal{K}$-FF Hamiltonian $H$, then we will need to find a $\mathcal{K}$-local Hamiltonian $H_{1}$ such that the null space of $H_{1}$ contains $V^{\prime}$, and for any state $|\phi\rangle \in V-V^{\prime},\left\langle\phi\left|H_{1}\right| \phi\right\rangle>0$. Then, for large-enough $t$, the $\mathcal{K}$-local Hamiltonian $t H_{0}+H_{1}$ will have $V^{\prime}$ as its ground-state space.

To demonstrate the application of these methods, we consider two examples. Our first example is the case of three qubits that we will discuss in Sec. VA. And the second example are those $W$-type states that we will discuss in Sec. V B.

\section{A. The three-qubit case}

It is well known that almost all three-qubit pure states are uniquely determined by their 2-RDMs, except those states which are local unitary (LU) equivalent to GHZ-type states $\alpha|000\rangle+\beta|111\rangle[5]$. That is, almost all three-qubit pure states are $\mathcal{K}^{\prime}$ correlated for

$$
\mathcal{K}^{\prime}=\{\{1,2\},\{2,3\},\{1,3\}\} .
$$

Here we will then find the $\mathcal{K}^{\prime}$-local Hamiltonian $H$ for all three-qubit states, starting from a $\mathcal{K}^{\prime}$-FF Hamiltonian and using the perturbation method, except for those states which are LU equivalent to GHZ-type states. Indeed, our method finds some $\mathcal{K}$-local Hamiltonians for these states, where

$$
\mathcal{K}=\{\{1,2\},\{2,3\}\} \subset \mathcal{K}^{\prime} .
$$

This means that indeed all three-qubit pure states are uniquely determined by their 2-RDMs of particles $\{1,2\}$ and $\{2,3\}$, except for those states which are LU equivalent to GHZ-type states. In other words, only two out of the three 2-RDMs are enough to uniquely determine these states, which is an improvement of the results given in [5].

Note that one of the standard forms for a three-qubit pure state up to LU transformation is [13]

$|\psi\rangle_{123}=\lambda_{0}|000\rangle+\lambda_{1}|100\rangle+\lambda_{2}|101\rangle+\lambda_{3}|110\rangle+\lambda_{4}|111\rangle$,

where $\lambda_{1}$ is complex, and $\lambda_{0}, \lambda_{2}, \lambda_{3}, \lambda_{4}$ are real.

We start from constructing a $\mathcal{K}$-FF Hamiltonian $H_{\mathcal{K}}$ for $\mathcal{K}=\{\{1,2\},\{2,3\}\}$, which contains $|\psi\rangle_{123}$ as a ground state. Define the space $\mathcal{S}$ as

$$
\mathcal{S}=\left[\operatorname{range}\left(\gamma_{\{1,2\}}\right) \otimes I_{\{3\}}\right] \cap\left[I_{\{1\}} \otimes \operatorname{range}\left(\gamma_{\{2,3\}}\right)\right],
$$

where $\gamma_{\{i, j\}}$ is the 2-RDM of $|\psi\rangle_{123}$ of particles $\{i, j\}$.

It is straightforward to show that $\mathcal{S}$ is always two dimensional for any entangled $|\psi\rangle_{123}$. That is, $|\psi\rangle_{123}$ cannot be written as a product of a single- and a two-qubit state. In this case, one always has

$\left|\psi^{\prime}\right\rangle_{123}=|1\rangle \otimes\left(\lambda_{2}|0\rangle+\lambda_{4}|1\rangle\right) \otimes\left(\lambda_{3}|0\rangle+\lambda_{4}|1\rangle\right) \in \mathcal{S}$.

That is, $\mathcal{S}$ always contains a product state $\left|\psi^{\prime}\right\rangle_{123}$ (see [14]).

We can then choose a $\mathcal{K}$-FF Hamiltonian

$$
H_{0}=H_{\{1,2\}}+H_{\{2,3\}},
$$


where $H_{\{i, j\}}$ is the projection onto the kernel of $\gamma_{\{i, j\}}$. Then $\mathcal{S}$ is the ground-state space of $H_{0}$, which is two dimensional and spanned by $|\psi\rangle_{123}$ and $\left|\psi^{\prime}\right\rangle_{123}$.

Now we need to find a perturbation $\mathcal{K}^{\prime}$-local Hamiltonian $H_{1}$ such that for large-enough $t$, the $\mathcal{K}^{\prime}$-local Hamiltonian $t H_{0}+H_{1}$ has $|\psi\rangle_{123}$ as its unique ground state. First, define

$$
\begin{aligned}
|\phi\rangle_{123} & =\lambda_{1}|100\rangle+\lambda_{2}|101\rangle+\lambda_{3}|110\rangle+\lambda_{4}|111\rangle \\
& =|1\rangle \otimes\left(\lambda_{1}|00\rangle+\lambda_{2}|01\rangle+\lambda_{3}|10\rangle+\lambda_{4}|11\rangle\right) \\
& =|1\rangle \otimes|\tilde{\psi}\rangle_{23} .
\end{aligned}
$$

For the generic case, $|\phi\rangle_{123}$ is linear independent of $\left|\psi^{\prime}\right\rangle_{123}$, which means

$$
\lambda_{1} \lambda_{4} \neq \lambda_{2} \lambda_{3}
$$

Now define

$$
\eta={ }_{123}\left\langle\psi^{\prime} \mid \phi\right\rangle_{123}=\lambda_{1} \lambda_{2} \lambda_{3}+\lambda_{2}^{2} \lambda_{4}+\lambda_{3}^{2} \lambda_{4}+\lambda_{4}^{3},
$$

and choose

$$
|\xi\rangle_{123}=\left|\psi^{\prime}\right\rangle_{123}-\eta^{*}|\psi\rangle_{123} .
$$

Note that

$$
{ }_{123}\langle\psi \mid \xi\rangle_{123}=0
$$

and $|\xi\rangle_{123}$ has a form

$$
|\xi\rangle_{123}=\alpha|000\rangle+\beta|1\rangle \otimes|\tilde{\xi}\rangle_{23},
$$

where $|\tilde{\xi}\rangle_{23}$ is a pure state of particles $\{2,3\}$ which is linear independent of $|\tilde{\psi}\rangle_{23}$ according to Eq. (21).

We can then choose a two-particle Hermitian operator $H_{\{2,3\}}^{\prime}$ acting on particles $\{2,3\}$ such that $|00\rangle_{23}$ and $|\tilde{\psi}\rangle_{23}$ span the kernel of $H_{\{2,3\}}^{\prime}$, hence ${ }_{23}\langle\tilde{\xi}|H| \tilde{\xi}\rangle_{23}=r \neq 0$. So the perturbation Hamiltonian $H_{1}$ can just be chosen as $H_{1}=H_{\{2,3\}}^{\prime}$ if $r>0$ or $H_{1}=-H_{\{2,3\}}^{\prime}$ if $r<0$. Then, for large-enough $t$, $t H_{0}+H_{1}$ has $|\psi\rangle_{123}$ as its unique ground state.

For the case in which $|\phi\rangle_{123}$ is linear dependent of $\left|\psi^{\prime}\right\rangle_{123}$, which in general means

$$
\lambda_{1} \lambda_{4}=\lambda_{2} \lambda_{3},
$$

we can also find $H_{1}$, unless $|\phi\rangle_{123}$ is LU equivalent to the GHZ-type state. Note that Eq. (26) indicates that $\lambda_{1}$ is real.

We can rewrite $|\psi\rangle_{123}$ as

$$
|\psi\rangle_{123}=\lambda_{0}|000\rangle+\lambda_{x y}|1 x y\rangle,
$$

where

$$
|x\rangle=x_{0}|0\rangle+x_{1}|1\rangle, \quad|y\rangle=y_{0}|0\rangle+y_{1}|1\rangle,
$$

with $x_{0}, x_{1}, y_{0}, y_{1}$ real, $x_{0}^{2}+x_{1}^{2}=1$, and $y_{0}^{2}+y_{1}^{2}=1$.

We know that $|1 x y\rangle$ is also in the ground-state space of $H_{0}$, so the ground-state space of $H_{0}$ is actually spanned by two orthogonal product states, $|000\rangle$ and $|1 x y\rangle$.

In general, when $|\psi\rangle_{123}$ is not LU equivalent to the GHZtype state, we have

$$
\langle 0 \mid x\rangle \neq 0 \text { or }\langle 0 \mid y\rangle \neq 0 .
$$

Without loss of generality, we assume $\langle 0 \mid y\rangle \neq 0$, that is, $y_{0} \neq 0$.

Now we need to find some Hamiltonian $H_{1}$ to "split" $|000\rangle$ and $|1 x y\rangle$ such that for large-enough $t$, the ground state of $t H_{0}+H_{1}$ could be uniquely $\left|\psi_{123}\right\rangle$, based on the perturbation method. We show this is always possible. Let

$D_{1}=\left(\begin{array}{cc}\frac{\lambda_{x y}}{\lambda_{0}} & 0 \\ 0 & \frac{\lambda_{0}}{\lambda_{x y}}\end{array}\right), \quad M_{2}=\left(\begin{array}{cc}x_{0} & x_{1} \\ x_{1} & -x_{0}\end{array}\right), \quad M_{3}=\left(\begin{array}{cc}y_{0} & y_{1} \\ y_{1} & -y_{0}\end{array}\right)$.

Then we have

$$
X_{1} D_{1} \otimes M_{2} \otimes M_{3}\left|\psi_{123}\right\rangle=\left|\psi_{123}\right\rangle,
$$

which gives

$$
X_{1} D_{1} \otimes M_{2} \otimes I_{3}\left|\psi_{123}\right\rangle=I_{1} \otimes I_{2} \otimes M_{3}\left|\psi_{123}\right\rangle,
$$

where $I_{j}$ is the identity operator acting on the $j$ th particle.

Now we can choose a two-particle operator,

$$
H_{1}^{\prime}=X_{1} D_{1} \otimes M_{2} \otimes I_{3}-I_{1} \otimes I_{2} \otimes M_{3} .
$$

Then, $\left|\psi_{123}\right\rangle$ is in the kernel of $H_{1}^{\prime}$, and $\left\langle 000\left|H_{1}^{\prime}\right| 000\right\rangle \neq 0$.

Let $\left|\psi^{\perp}\right\rangle_{123}=\lambda_{x y}|000\rangle-\lambda_{0}|1 x y\rangle$, and ${ }_{123}\left\langle\psi^{\perp}\left|H_{1}^{\prime}\right| \psi^{\perp}\right\rangle_{123}=$ $r \neq 0$; then the perturbation Hamiltonian $H_{1}$ can just be chosen as $H_{1}=H_{1}^{\prime}$ if $r>0$ or $H_{1}=-H_{1}^{\prime}$ if $r<0$. Then, for largeenough $t, t H_{0}+H_{1}$ has $|\psi\rangle_{123}$ as its unique ground state.

A similar procedure works if $\langle 0 \mid y\rangle=0$ but $\langle 0 \mid x\rangle \neq 0$. The procedure will fail to result in having $|\psi\rangle_{123}$ as the unique ground state $t H_{0}+H_{1}$ for any $t$ if both $\langle 0 \mid x\rangle \neq 0$ and $\langle 0 \mid y\rangle \neq 0$. In that case, one will have ${ }_{123}\left\langle\psi^{\perp}\left|H_{1}^{\prime}\right| \psi^{\perp}\right\rangle_{123}=0$. And using a properly chosen $t$, one can have $|\psi\rangle_{123}$ as the nondegenerate first excited state $t H_{0}+H_{1}$ [15].

To summarize, we have found the $\mathcal{K}$-local Hamiltonian for all three-qubit pure states for $\mathcal{K}=\{\{1,2\},\{2,3\}\}$, except for those states which are LU equivalent to GHZ-type states. By Observation 1, our result also shows that all three-qubit pure states are uniquely determined by their 2-RDMs of particles $\{1,2\}$ and $\{2,3\}$, except for those states which are LU equivalent to GHZ-type states.

\section{B. The $W$-type states}

In this section, we discuss the $n$-qubit $W$-type states $|W(n)\rangle_{\text {type }}$,

$$
|W(n)\rangle_{\text {type }}=\sum_{i=1}^{n} a_{s}\left|\mathbf{r}_{i}\right\rangle
$$

where $\mathbf{r}_{i}$ is the $n$-bit strings with the $i$ th coordinate 1 and all the other coordinates $0, a_{i} \neq 0$ and $\sum_{i=1}^{n}\left|a_{i}\right|^{2}=1$.

It is known that $|W(n)\rangle_{\text {type }}$ is uniquely determine by its 2-RDM [7]. Furthermore, any $n-1$ out of the $\left(\begin{array}{l}n \\ 2\end{array}\right) 2-\mathrm{RDMs}$ is sufficient to uniquely determine $|W(n)\rangle_{\text {type }}$, so we can actually put the $n$ qubit on a one-dimensional chain and consider only the 2-RDMs of all the nearest-neighbor pairs. More precisely, let

$$
\mathcal{K}=\{\{1,2\}, \ldots,\{n-1, n\}\} .
$$

Then, the $W$-type states are $\mathcal{K}$ correlated.

Here we discuss how to find the $\mathcal{K}$-local Hamiltonian whose unique ground state is a given $W$-type state. We start from the three-qubit case. In Sec. V A, we have already solved this problem for all three-qubit pure states. Here we reexamine the $W$ state case so that we understand how to generalize it to the 
general $n$-qubit case. We start from the fact that the three-qubit $W$-type state can be written as

$$
|W(3)\rangle_{\mathrm{type}}=a_{1}|001\rangle+a_{2}|010\rangle+a_{3}|100\rangle,
$$

and observe that

$$
\begin{aligned}
& {\left[I_{\{1\}} \otimes \operatorname{range}\left(\gamma_{\{2,3\}}\right)\right] \cap\left[\operatorname{range}\left(\gamma_{\{1,2\}}\right) \otimes I_{\{3\}}\right]} \\
& \quad=\operatorname{span}\left\{|W\rangle_{\text {type }},|000\rangle\right\},
\end{aligned}
$$

and

$$
\langle 000 \mid W(3)\rangle_{\text {type }}=0 .
$$

We can first choose a $\mathcal{K}$-FF Hamiltonian

$$
H_{0}=H_{\{1,2\}}+H_{\{2,3\}}
$$

for $\mathcal{K}=\{\{1,2\},\{2,3\}\}$. Here, $H_{\{i, j\}}$ is the projection onto the kernel of $\gamma_{\{i, j\}}$. Then, $\left\{|W(3)\rangle_{t},|000\rangle\right\}$ spans the twodimensional ground-state space of $H_{0}$.

We can then choose

$$
H_{1}=-Z_{1}-Z_{2}-Z_{3} .
$$

For a large-enough $t,|W(3)\rangle_{\text {type }}$ is the unique ground state of $t H_{0}+H_{1}$.

Now we take a look at the special case where $a_{1}=a_{2}=a_{3}$, in which $|W(3)\rangle_{\text {type }}$ becomes the three-qubit $W$ state $|W(3)\rangle$, where

$$
|W(3)\rangle=\frac{1}{\sqrt{3}}(|001\rangle+|010\rangle+|100\rangle) .
$$

Now the $\mathcal{K}$-FF Hamiltonian $H_{0}$ given in Eq. (38) has a twodimensional ground-state space spanned by $\{|W(3)\rangle,|000\rangle\}$.

Note that now both $H_{\{1,2\}}$ and $H_{\{2,3\}}$ are projections onto the space spanned by

$$
|\alpha\rangle=|11\rangle, \quad|\beta\rangle=\frac{1}{\sqrt{2}}(|01\rangle-|10\rangle),
$$

thus $H_{\{1,2\}}$ can be written as

$$
H_{\{1,2\}}=p_{\alpha}|\alpha\rangle\left\langle\alpha\left|+p_{\beta}\right| \beta\right\rangle\langle\beta|,
$$

where $p_{\alpha}, p_{\beta}>0$.

In terms of Pauli operators, $H_{\{1,2\}}$ has a form

$$
\begin{aligned}
H_{\{1,2\}}=- & p_{\alpha}\left(X_{1} X_{2}+Y_{1} Y_{2}\right) \\
& +\left(p_{\beta}-p_{\alpha}\right) Z_{1} Z_{2}-p_{\beta}\left(Z_{1}+Z_{2}\right) .
\end{aligned}
$$

And a similar form holds for $H_{\{2,3\}}$.

This form of $H_{\{i, j\}}$ can be generalized to the $n$-qubit case. To see this, note that

$$
\bigcap_{i} \operatorname{range}\left(\gamma_{\{i, i+1\}}\right) \otimes I_{\{\overline{i, i+1}\}}=\{|W(n)\rangle,|00 \ldots 0\rangle\} .
$$

Now we can choose $H_{0}=\sum_{i} H_{\{i, i+1\}}$,

$$
\begin{aligned}
H_{\{i, i+1\}}= & -p_{\alpha}\left(X_{i} X_{i+1}+Y_{i} Y_{i+1}\right) \\
& +\left(p_{\beta}-p_{\alpha}\right) Z_{i} Z_{i+1}-p_{\beta}\left(Z_{i}+Z_{i+1}\right),
\end{aligned}
$$

and $H_{1}=-\sum_{i} Z_{i}$. Then for a large-enough $t$, the $\mathcal{K}$-local Hamiltonian $H_{\mathcal{K}}=t H_{0}+H_{1}$ has the $n$-qubit $W$ state $|W(n)\rangle$ as its unique ground state.

If we take a periodic boundary condition instead of a chain, that is, choose

$$
\mathcal{K}^{\prime}=\{\{1,2\},\{2,3\}, \ldots,\{n-1, n\},\{n, 1\}\},
$$

then for a small enough $\epsilon$, the $\mathcal{K}^{\prime}$-local Hamiltonian $H_{w}$ of which $|W(n)\rangle$ is a unique ground state can be written as

$$
\begin{aligned}
H_{w}= & -\sum_{i, i+1}\left[p_{\alpha} X_{i} X_{i+1}+p_{\alpha} Y_{i} Y_{i+1}+\left(p_{\alpha}-p_{\beta}\right) Z_{i} Z_{i+1}\right] \\
& -\sum_{i}(2 \beta-\epsilon) Z_{i} .
\end{aligned}
$$

Actually, $\mathcal{H}_{w}$ has a recognized physical meaning, as it is a famous spin model called the "Heisenberg $X X Z$ model," where we also have a term of the external magnetic field, which is given by the second sum term in $\mathcal{H}_{w}$. This model is extensively studied in the literature; for instance, see [16] and references therein.

Note that our results are consistent with those obtained in [17], where a special case $p_{\alpha}=p_{\beta}$ is considered, so $\mathcal{H}_{w}$ is reduced to a Heisenberg $X X$ chain in a transversal magnetic field. We observe that although for different values of $p_{\alpha}$ and $p_{\beta}$, the ground state could be all uniquely $|W(n)\rangle$, the Hamiltonians $\mathcal{H}_{w}$ do have different spectrums, and hence are different Hamiltonians.

\section{HAMILTONIANS FROM THERMODYNAMICAL ENSEMBLES}

In this section, we discuss a general method to determine whether a given state space $V$ is $\mathcal{K}$ correlated; if so, we find the $\mathcal{K}$-local Hamiltonian such that its ground-state space is $V$. Our approach is based on the viewpoint of thermodynamical ensembles.

For a given space $V$, we introduce

$$
\rho(p)=p \frac{I}{D}+(1-p) \rho_{V},
$$

where $I$ is the identity operator acting on the Hilbert space $\mathcal{H}$ of the $n$-particle system with a finite dimension $D$, and $\rho_{V}$ is the maximally mixed state of $V$. Obviously, $\rho(0)=\rho_{V}$.

As the state $\rho(p)$ is of full rank for $p \in[1,0), \tilde{\rho}_{\mathcal{K}}(p)$, as given by Eq. (8), can be written in an exponential form [4]

$$
\tilde{\rho}_{\mathcal{K}}(p)=\frac{\exp \left[-\tilde{H}_{\mathcal{K}}(p)\right]}{\operatorname{Tr} \exp \left[-\tilde{H}_{\mathcal{K}}(p)\right]},
$$

where $\vec{R}_{\mathcal{K}}\left(\tilde{\rho}_{\mathcal{K}}(p)\right)=\vec{R}_{\mathcal{K}}(\rho(p))$, and the Hermitian operator $\tilde{H}_{\mathcal{K}}(p)$ is $\mathcal{K}$ local. And, indeed, such an exponential form is unique [18].

The key observation here is that $\tilde{\rho}_{\mathcal{K}}(p)$ can be viewed as a thermal equilibrium state corresponding to the $\mathcal{K}$-local Hamiltonian $H_{\mathcal{K}}(p)$ : we can define $\beta(p) H_{\mathcal{K}}(p)=\tilde{H}_{\mathcal{K}}(p)$ with $\beta(p)$, which is a positive constant that is inversely proportional to temperature.

Note that the maximally mixed state $\rho_{V}$ of $V$ is an equal weight mixture of orthonormal pure states, which span $V$. As $\rho_{V}$ is $\mathcal{K}$ correlated, we have

$$
\lim _{p \rightarrow 0} \tilde{\rho}_{\mathcal{K}}(p)=\rho_{V}
$$

according to the continuity principle given in $[4,18]$. Equation (50) then implies that $\rho_{V}$ is the equal weight mixture of the ground states of $H_{\mathcal{K}}(0)$, and the corresponding temperature goes to 0 , i.e., $\lim _{p \rightarrow 0} \beta(p)=+\infty$. 
Note that the continuity principle discussed in $[4,18]$ is an argument, not a rigorous proof. And this method definitely fails for those $\mathcal{K}$-correlated spaces, which is similar to point $A$ in Fig. 1. However, this viewpoint of the thermal equilibrium ensemble gives a good physical intuition to understand Observation 1.

One numerical method to find $\tilde{H}_{\mathcal{K}}(p)$ for $p \in[1,0)$ can be developed based on the discussion in [19]. The idea is that if the continuity principle is valid, then when $p$ is arbitrarily close to one, the ground-state space of $\tilde{H}_{\mathcal{K}}(p)$ will be also arbitrarily close to $V$.

As an example to test our numerical method, consider the following four-qubit state:

$$
\left|\psi_{1}\right\rangle=\frac{1}{2}(|0000\rangle+|0101\rangle+|1000\rangle+|1110\rangle) .
$$

Our numerical method shows that there exists a Hamiltonian containing only one- and two-particle interaction terms, such that $\left|\psi_{1}\right\rangle$ is the unique ground state. This Hamiltonian can be given by $p=0.0001$; that is,

$$
\begin{aligned}
\tilde{H}(0.0001)= & -3.2390 Z_{4}+4.2001 X_{3} X_{4}+4.2001 Y_{3} Y_{4} \\
& -3.2390 Z_{3}-0.5912 Z_{3} Z_{4}-6.4827 X_{2} X_{4} \\
& -6.4827 X_{2} X_{3}+6.4827 Y_{2} Y_{4}+6.4827 Y_{2} Y_{3} \\
& +6.7571 Z_{2}+1.5227 Z_{2} Z_{4}+1.5227 Z_{2} Z_{3} \\
& -4.2950 X_{1}-2.4012 X_{1} Z_{4}-2.4012 X_{1} Z_{3} \\
& -8.8603 X_{1} Z_{2}+4.5280 Z_{1} Z_{4}-4.5280 Z_{1} Z_{3},
\end{aligned}
$$

and one can readily check that $\left|\psi_{1}\right\rangle$ is the unique ground state of $\tilde{H}(0.0001)$. By Observation $1,\left|\psi_{1}\right\rangle$ is then $\mathcal{K}\left(\psi_{1}\right)$ correlated for

$$
\mathcal{K}\left(\psi_{1}\right)=\{\{1,2\},\{2,3\},\{1,3\},\{3,4\},\{2,4\},\{1,4\}\} .
$$

This method also allows us to determine whether a given space $V$ is $\mathcal{K}$ correlated or not. If the method returns a $\mathcal{K}$-local Hamiltonian $H(p)$ with $p$ sufficiently small, whose ground-state space is larger than $V$, then $V$ is not $\mathcal{K}$ correlated. Otherwise, it returns exactly $V$.

As an example, consider the following state:

$$
\left|\psi_{2}\right\rangle=\frac{1}{2}(|0000\rangle+|1011\rangle+|1101\rangle+|1110\rangle) \text {. }
$$

Our numerical method shows that there does not exist a Hamiltonian containing only one- and two-particle interaction terms, such that $\left|\psi_{2}\right\rangle$ is the unique ground state. Indeed, the state

$$
\left|\psi_{2}^{\prime}\right\rangle=\frac{1}{2}(-|0000\rangle+|1011\rangle+|1101\rangle+|1110\rangle)
$$

has the same $\mathcal{K}$ projection as that of $\left|\psi_{2}\right\rangle$.

One would expect that our numerical method cannot be efficient in general. Indeed, even in practice, the Hamiltonians that we are interested in mainly involve only one- and two-particle interaction terms associated with certain lattice geometry; the complexity of our numerical method grows superexponential with the system size $n$. Therefore, for each special case considered, one usually needs to combine this method with some other techniques.

Here we introduce a method of subsystems to reduce the complexity of the above numerical method for some specific cases, based on the discussion of frustration-free systems given in Sec. V. That is, in some cases, we can start from a $\mathcal{K}$-FF
Hamiltonian and look at the subsystems of each term of the $\mathcal{K}$ FF Hamiltonian. The advantage of this method of subsystems is that one can reduce the total dimension of the Hilbert space that one needs to calculate the $\mathcal{K}$-local Hamiltonians by using some frustration-free properties of the quantum space $V$.

Recall that a $\mathcal{K}$-FF Hamiltonian is $\mathcal{K}$ local. Denote $P\left(K_{i}\right)$ as the power set of $K_{i}$ for each $K_{i} \in \mathcal{K}$. We then define

$$
\mathcal{K}^{\prime}=\bigcup_{i} \mathcal{K}_{i}^{\prime}
$$

where each $\mathcal{K}_{i}^{\prime}$ is a subset of $P\left(K_{i}\right)$. In practice, we will be interested in some pattern $\mathcal{K}^{\prime}$ with $\left|K_{j}^{\prime}\right|=k^{\prime}$ for $K_{j}^{\prime} \in \mathcal{K}^{\prime}$, where $k^{\prime}<k$. In other words, the $\mathcal{K}$-FF Hamiltonian contains $k$-particle interactions, but the $\mathcal{K}^{\prime}$-local Hamiltonian that we want to find contains only $k^{\prime}<k$-particle interactions.

The following observation provides a method of finding a $\mathcal{K}^{\prime}$-local Hamiltonian for the ground-state space $V$ of a $\mathcal{K}$-FF Hamiltonian.

Observation 5. Given a space $V$, which is the groundstate space of a $\mathcal{K}$-FF Hamiltonian, if for any $K_{i} \in \mathcal{K}$, range $\left[\rho_{V}\left(K_{i}\right)\right]$ is $\mathcal{K}_{i}^{\prime}$ correlated, then $V$ is $\mathcal{K}^{\prime}$ correlated.

To see how this observation works, for each $K_{i}$, range $\left[\rho_{V}\left(K_{i}\right)\right]$ is $\mathcal{K}_{i}^{\prime}$ correlated, so one can find a $\mathcal{K}_{i}^{\prime}$-local Hamiltonian $H_{\mathcal{K}_{i}^{\prime}}$ which has range $\left[\rho_{V}\left(K_{i}\right)\right]$ as its ground-state space. However, these spaces of range $\left[\rho_{V}\left(K_{i}\right)\right]$ determine $V$, i.e., $\bigcap_{i} \operatorname{range}\left[\rho_{V}\left(K_{i}\right)\right]=V$, so the Hamiltonian $\sum_{i} H_{\mathcal{K}_{i}^{\prime}}$ has $V$ as its ground-state space.

As an example, consider the state $\left|\psi_{1}\right\rangle$ given in Eq. (51). It is straightforward to show that $\left|\psi_{1}\right\rangle$ is the unique ground state of a $\mathcal{K}$-FF Hamiltonian for $\mathcal{K}=\{\{1,2,3\},\{2,3,4\}\}$. However, this will give us a nonpractical Hamiltonian, which involves three-particle interactions.

Note that the space $V_{\{1,2,3\}}=$ range $\left[\left|\psi_{1}\right\rangle\left\langle\psi_{1}\right|(\{1,2,3\})\right]$ is spanned by

$$
V_{\{1,2,3\}}=\operatorname{span}\{|000\rangle+|110\rangle+|111\rangle,|010\rangle\},
$$

and the space $V_{\{2,3,4\}}=\operatorname{ker}\left[\left|\psi_{1}\right\rangle\left\langle\psi_{1}\right|(\{2,3,4\})\right]^{\perp}$ is spanned by

$$
V_{\{2,3,4\}}=\operatorname{span}\{|000\rangle+|101\rangle,|000\rangle+|110\rangle\} .
$$

We can now use our numerical method to further show that $V_{\{1,2,3\}}$ is $\{\{1,2\},\{2,3\},\{1,3\}\}$ correlated, and $V_{\{2,3,4\}}$ is $\{\{2,3\},\{3,4\},\{2,4\}\}$ correlated. Therefore, by Observation 5, $\left|\psi_{1}\right\rangle$ is $\mathcal{K}^{\prime}\left(\psi_{1}\right)$ correlated for

$$
\mathcal{K}^{\prime}\left(\psi_{1}\right)=\{\{1,2\},\{2,3\},\{1,3\},\{3,4\},\{2,4\}\} .
$$

In this example, we use the method of subsystems to reduce the calculation in our algorithm for a $n=4$ state to two $n=3$ spaces. One could expect that for a larger system with ground-state space of some local frustration-free Hamiltonians involving at most $k$-particle interactions, this method of subsystems may further reduce the calculation in our numerical method from a large $n$ to some small number $k$. Moreover, recalling Eq. (52), we actually have $\mathcal{K}^{\prime}\left(\psi_{1}\right) \subset \mathcal{K}\left(\psi_{1}\right)$, so the result obtained by this method of subsystems gives a slightly simpler interaction pattern of the Hamiltonian.

Finally, as a remark, note that the reverse of Observation 5 is not true, as the space $V_{\{1,3,4\}}=\operatorname{range}\left[\left|\psi_{1}\right\rangle\left\langle\psi_{1}\right|(\{1,3,4\})\right]$, 
spanned by

$$
V_{\{1,3,4\}}=\operatorname{span}\{|000\rangle+|100\rangle,|001\rangle+|110\rangle\},
$$

is not $\{\{1,2\},\{3,4\},\{1,4\}\}$ correlated.

\section{CONCLUSION AND DISCUSSION}

In this paper, we raised an interesting question regarding "from ground states to local Hamiltonians." That is, for a given space $V$, one would like to know whether $V$ can be the groundstate space of some $k$-local Hamiltonian $H=\sum_{i} H_{i}$, which contains only certain terms of $k$-particle interactions, and, if such a $k$-local Hamiltonian exists, how to find it. As a starting point, it turns out that a natural necessary condition for a space $V$ to be the ground-state space of some local Hamiltonian with a given interaction pattern is that the maximally mixed state supported on $V$ is uniquely determined by its reduced density matrices associated with the given pattern based on the principle of maximum entropy. This simple observation builds an interesting link between correlations of quantum states and the ground-state space of local Hamiltonians.

We have introduced the concept of $\mathcal{K}$-correlated spaces and explained its physical and geometric meaning. By introducing the concept of $\mathcal{K}$-local Hamiltonians, which describe local Hamiltonians with given interaction patterns in a more formal way, the necessary condition that a space $V$ is the ground-state space of some $\mathcal{K}$-local Hamiltonian is that $V$ is $\mathcal{K}$ correlated. However, this $\mathcal{K}$ correlatedness of a space $V$ does not guarantee that $V$ can be the exact ground-state space of some $\mathcal{K}$-local Hamiltonian. To understand why this necessary condition may not be sufficient and when this problem could possibly happen, we link the spaces satisfying this necessary condition to faces of the convex body of the local reduced states. Based on this understanding of convex geometry, we then further discuss some methods for constructing the corresponding $\mathcal{K}$-local Hamiltonians, mainly from physical points of view, including constructions related to perturbation methods, local frustration-free Hamiltonians, as well as thermodynamical ensembles.

The perturbation method, combined with the method based on the frustration-free systems, allows us to identify the $\mathcal{K}$-local Hamiltonians for all three-qubit states for $\mathcal{K}=$ $\{\{1,2\},\{2,3\}\}$, except those states which are LU equivalent to GHZ-type states. In other words, all of the extreme points on the corresponding convex body are exposed in this case. Our result then shows that only two out of the three 2-RDMs are enough to uniquely determine a three-qubit pure state unless the state is LU equivalent to a GHZ-type state, which is an improvement of the result given in [5]. We also find the $X X$-type Hamiltonians for $W$ states, which are identified in [16] from other methods.

The method based on an idea of thermal ensembles provides an alternative and a more physical understanding of the relationship between $\mathcal{K}$-correlated spaces and $\mathcal{K}$-local Hamiltonians, as well as a numerical method of finding such a $\mathcal{K}$-local Hamiltonian. This numerical method is based on the continuity principle discussed in $[4,18,19]$. And combined with a method of subsystems which is related to local frustration-free Hamiltonians, the computational cost may be reduced for some special physical systems.
One would think the direct way of dealing with the problem of finding the $\mathcal{K}$-local Hamiltonian for a given $\mathcal{K}$-correlated space is through a general algorithmic viewpoint. Indeed, this problem can be straightforwardly formulated in terms of a semidefinite programming [20], which can be used to numerically solve this problem.

However, in general, finding a $\mathcal{K}$-local Hamiltonian with a given $\mathcal{K}$-correlated space $V$ as its exact ground-state space is a very hard problem. Theoretically, none of these methods could work if some $\mathcal{K}$-correlated spaces have a similar property as the point $A$ in Fig. 1. So it is highly desired to find a theoretical characterization of those $\mathcal{K}$-correlated spaces that cannot be the ground-state space of any $\mathcal{K}$-local Hamiltonian, or find a proof to show that such $\mathcal{K}$-correlated spaces do not really exist.

Also, even if such a $\mathcal{K}$-local Hamiltonian does exist for a $\mathcal{K}$-correlated space, it is expected that all of the methods and algorithms we have discussed here are not efficient for the general case. Indeed, one can only expect that each method works well in certain special cases, as those examples discussed. Future work will be toward identifying better methods and algorithms for special situations, especially for $\mathcal{K}$-correlated spaces which are of interest to quantum information processing, for instance those resource states for one-way quantum computing [21]. On the other hand, one would also like to develop methods to identify whether a space is $\mathcal{K}$ correlated, even without finding the corresponding $\mathcal{K}$-local Hamiltonian.

We hope our work sheds light on the study of the relationship between correlations of quantum states and ground-state spaces of local Hamiltonians, and thus further link the research in both quantum information science and many-body physics.

\section{ACKNOWLEDGMENTS}

We thank Mary Beth Ruskai, Runyao Duan, and Salman Beige for helpful discussions. J.C. is supported by NSERC. Z.J. acknowledges support from ARO and NSF of China (Grants No. 60736011 and No. 60721061). B.Z. is supported by NSERC and CIFAR. D.Z. is supported by NSF of China (Grants No. 10975181 and No. 11175247) and NKBRSF of China (Grant No. 2012CB922104).

\section{APPENDIX: PROOF OF OBSERVATION 1}

To prove the equivalences in Observation 1, we need the following two lemma:

Lemma 1. If range $\left(\rho_{1}\right) \nsubseteq \operatorname{range}\left(\rho_{0}\right)$, then there exists $x^{\star} \in$ $(0,1)$ such that

$$
S\left(\left(1-x^{\star}\right) \rho_{0}+x^{\star} \rho_{1}\right)>S\left(\rho_{0}\right) .
$$

Proof. For simplicity, let $\rho_{x}=(1-x) \rho_{0}+x \rho_{1}$. A direct calculation gives

$$
\begin{aligned}
S\left(\rho_{x}\right)-S\left(\rho_{0}\right)= & x\left(S\left(\rho_{1}\right)-S\left(\rho_{0}\right)+S\left(\rho_{1} \| \rho_{x}\right)\right) \\
& +(1-x) S\left(\rho_{0} \| \rho_{x}\right) .
\end{aligned}
$$

The assumption, range $\left(\rho_{1}\right) \nsubseteq \operatorname{range}\left(\rho_{0}\right)$, implies that $S\left(\rho_{1} \| \rho_{x}\right)$ can be made arbitrarily large by choosing $x$ close to 0 . Therefore, we can find a $x^{\star} \in(0,1)$ satisfying $S\left(\rho_{1} \| \rho_{x^{\star}}\right)>$ $S\left(\rho_{0}\right)-S\left(\rho_{1}\right)$. As both terms of the above equation are positive for $x^{\star}$, we have $S\left(\rho_{x^{\star}}\right)>S\left(\rho_{0}\right)$. 
Lemma 2 . For any quantum state $\rho, \operatorname{range}(\rho) \subseteq \operatorname{range}\left(\tilde{\rho}_{k}\right)$.

Proof. If range $(\rho) \nsubseteq \operatorname{range}\left(\tilde{\rho}_{k}\right)$, then Lemma 1 guarantees that there is some number $p \in(0,1)$, such that $(1-p) \tilde{\rho}_{k}+p \rho$ will have larger entropy than $\tilde{\rho}_{k}$ has. This is a contradiction to the definition of $\tilde{\rho}_{k}$.

We are now ready to show Observation 1 . We will need to show that if $V=\operatorname{range}\left(\rho_{V}\right)$, where $\rho_{V}$ is $\mathcal{K}$ correlated, then for any $\sigma$ supported on $V$, any $\sigma^{\prime} \in A_{\mathcal{K}}(\sigma)$ is also supported on $V$.
As range $(\sigma) \subseteq \operatorname{range}(\rho)$, we can write $\rho=(1-\epsilon) \sigma^{\prime \prime}+\epsilon \sigma$ for some small number $\epsilon$, and $\operatorname{range}\left(\sigma^{\prime \prime}\right) \subseteq V$. Introduce a new state

$$
\hat{\rho}=(1-\epsilon) \sigma^{\prime \prime}+\epsilon \sigma^{\prime} .
$$

It is obvious that $\hat{\rho} \in A_{\mathcal{K}}(\rho)$, therefore $\operatorname{range}(\hat{\rho}) \subseteq \operatorname{range}(\rho)$ by Lemma 2 , and $\operatorname{range}\left(\sigma^{\prime}\right) \subseteq \operatorname{range}(\hat{\rho}) \subseteq \operatorname{range}(\rho)=V$, where the first inclusion follows from Eq. (A1).
[1] M. Nielsen and I. Chuang, Quantum Computation and Quantum Information (Cambridge University Press, Cambridge, UK, 2000).

[2] E. T. Jaynes, Phys. Rev. 106, 620 (1957).

[3] N. Linden and W. K. Wootters, Phys. Rev. Lett. 89, 277906 (2002).

[4] D. L. Zhou, Phys. Rev. Lett. 101, 180505 (2008).

[5] N. Linden, S. Popescu, and W. K. Wootters, Phys. Rev. Lett. 89, 207901 (2002).

[6] N. S. Jones and N. Linden, Phys. Rev. A 71, 012324 (2005).

[7] P. Parashar and S. Rana, Phys. Rev. A 80, 012319 (2009).

[8] S. N. Walck and D. W. Lyons, Phys. Rev. Lett. 100, 050501 (2008).

[9] F. F. Verstraete and J. I. Cirac, Phys. Rev. B 73, 094423 (2006).

[10] M. Fannes, B. Nachtergaele, and R. F. Werner, Commun. Math. Phys. 144, 443 (1992).
[11] R. T. Rockafellar, Convex Analysis (Princeton University Press, Princeton, NJ, 1996).

[12] Y. K. Liu, Proc. RANDOM, 438 (2006).

[13] A. Acin, A. Andrianov, E. Jane, and R.Tarrach, J. Phys. A: Math. Gen. 34, 6725 (2001).

[14] J. Chen, X. Chen, R. Duan, Z. Ji, and B. Zeng, Phys. Rev. A 83, 050301 (2011), arXiv:1004.3787 [quant-ph].

[15] J. Chen, Z. Ji, Z. Wei, and B. Zeng, Phys. Rev. A 85, 040303 (2012).

[16] T. Koma and B. Nachtergaele, Adv. Theor. Math. Phys. 2, 533 (1998).

[17] D. Bruß, N. Datta, A. Ekert, L. C. Kwek, and C. Macchiavello, Phys. Rev. A 72, 014301 (2005).

[18] D. L. Zhou, Phys. Rev. A 80, 022113 (2009).

[19] D. L. Zhou, arXiv:0909.3700.

[20] S. Boyd and L. Vandenberghe, Convex Optimization (Cambridge University Press, Cambridge, UK, 2004).

[21] D. Gross and J. Eisert, Phys. Rev. Lett. 98, 220503 (2007). 